\title{
Growth and Thyroid Function in Children With In Utero Exposure to Dioxin: A 5-Year Follow-Up Study
}

\author{
PEN-HUA SU, JIA-YUH CHEN, JEIN-WEN CHEN, AND SHU-LI WANG \\ Department of Pediatrics [P.-H.S., J.-Y.C.], Chung Shan Medical University Hospital, School of Medicine [P.-H.S., J.-Y.C.], and \\ Department of Public Health [S.-L.W.], Chung Shan Medical University, Taichung 402, Taiwan; Division of Environmental Health and \\ Occupational Medicine [J.-W.C., S.-L.W.], National Health Research Institutes, Zhunan 350, Taiwan
}

\begin{abstract}
Because placental polychlorinated dibenzo-p-dioxins, dibenzofurans (PCDD/Fs) levels are associated with decreased free thyroxine $\left(\mathrm{FT}_{4}\right)$ and thyroid stimulating hormone (TSH) in neonates, we assessed development by gender and maternal PCDD/Fs exposure at years 2 and 5 in 92 mother and newborn pairs. RIA quantified thyroid, sex, and growth hormones. Of 200 subjects followed up from November 2000, 136 and 149 were observed at year 2 and year 5, respectively. PCDD/Fs exposure levels were low $(n=35)$ or high $(n=$ $35)$ in 70 subjects at year 2 , low $(n=21)$ or high $(n=20)$ in 41 at year 5. Height, weight, BMI, and head circumference were significantly higher in males, chest girth significantly higher in females at year 2. Significantly, more girls had higher bone age (BA) and chronological age $(\mathrm{CA})$ at both times. Height, weight, $\mathrm{FT}_{4} \times \mathrm{TSH}$, and transthyretin (TTR) at year 2; and height, triiodothyronine, and IGF-1 at year 5 differed significantly by PCDD/Fs level. In females, height, weight, CA, $\mathrm{BA}$, and thyroid hormones differed significantly at year 2. In males, $\mathrm{FT}_{4} \times \mathrm{TSH}$ at year 2 and IGF-1 at year 5 were significantly higher in the high $\mathrm{PCDD} / \mathrm{Fs}$ group. In utero exposure to $\mathrm{PCDD} / \mathrm{Fs}$ differentially affects growth and hormone levels in male and female preschool children. (Pediatr Res 67: 205-210, 2010)
\end{abstract}

$\mathrm{D}$ ioxins [polychlorinated dibenzo-p-dioxins, dibenzofurans $(\mathrm{PCDD} / \mathrm{Fs})]$ are highly toxic environmental contaminants, which are lipophilic and resistant to biodegradation. These compounds enter the human body through the food chain and finally accumulate in adipose tissue (1). A series of deleterious health effects are thought to be associated with organochlorine exposure. These include fetal and infant growth retardation and developmental abnormalities $(2,3)$, altered reproductive capacity $(4,5)$, carcinogenicity $(6,7)$, cognitive deficits (8), and impaired thyroid function (9). It has been confirmed that $P C D D / F s$ can be transferred from mother to fetus via the placenta (10) and from mother to infant via breast milk (11). The effects of the endocrine disruption during these critical stages of early development might persist long after birth.

Similar to other industrialized and developing countries, Taiwan is confronted with the problem of environmental $P C D D / F s$ contamination. It has been reported that high concentrations of $P C D D / F$ s are more prevalent in central Taiwan

Received June 3, 2009; accepted October 13, 2009.

Correspondence: Shu-Li Wang, Ph.D., Division of Environmental Health and Occupational Medicine, National Health Research Institutes, 35 Keyan Road, Zhunan, Miaoli County 350, Taiwan; e-mail: slwang@nhri.org.tw, jen@csh.org.tw

This work was supported by grants EO-097-PP-05 and EO-098-PP-03 from the National Health Research Institutes. than other areas of Taiwan (12). In a previous study, we examined the association between in utero exposure to $P C D D / F$ s/polychlorinated biphenyls (PCBs) and altered thyroid and growth hormone concentrations in newborns from central Taiwan (13), we found that after adjusting for other dioxins and PCB congeners in neonates from the general population, the multiplied concentration of free thyroxine $\left(\mathrm{FT}_{4}\right) \times$ thyroid stimulating hormone $(\mathrm{TSH})$ decreased with increasing levels of non-ortho PCBs in the placenta. In the same study, we also noted a significant and positive association between thyroxine $\left(\mathrm{T}_{4}\right)$ and thyroid-binding globulin concentrations with levels of PCDD/Fs (13).

Because thyroid hormones are crucial for growth and development, thyroid dysfunction in early infancy/childhood may adversely impair these processes and have serious longterm consequences. Hence, we conducted follow-up assessments at preschool ages for the cohort of neonates in our previous study (13). Because a previous report found that schoolchildren (aged 7.5-15 y) whose serum level of PCBs was two to four times higher than normal children had no significant impairment of thyroid function (14), the focus of this study was the relationship between in utero exposure to $\mathrm{PCDD} / \mathrm{Fs}$ and thyroid function and growth hormones. The objectives of the follow-up study were to examine possible abnormalities in growth and development, bone age (BA) and growth, and hormones related to neurodevelopment in children aged between 2 and $3 \mathrm{y}$ and 5 and $6 \mathrm{y}$ who were exposed to differing levels of PCDD/Fs in utero. Specifically, we made comparisons between children who were exposed to low and high levels of PCDD/Fs.

\section{MATERIALS AND METHODS}

Subjects. A total of 92 mother and newborn pairs were recruited from the general population in central Taiwan. We consecutively followed up the subjects $(n=200)$ of our previous study (13) from November 2000 to February 2007. We studied the subjects at $2-3$ y of age $(n=188$, during $2003)$ and at $5-6$ y $(n=149$, during 2006-2007). These groups are referred to as year 2 and year 5 , respectively. Children were also classified into low exposure and high exposure groups by the cutoff value $15 \mathrm{pg}-\mathrm{TEQ} / \mathrm{g}$ lipid based on the mother's PCDD/Fs TEQ levels. This study was approved by the

Abbreviations: BA, bone age; CA, chronological age; $\mathbf{E}_{\mathbf{2}}$, estradiol; $\mathbf{F T}_{\mathbf{4}}$, free thyroxine; IGFBP-3, insulin-like growth factor binding protein 3; PCBs, polychlorinated biphenyls; PCDD/Fs, polychlorinated dibenzo-p-dioxins, dibenzofurans; $\mathbf{T}_{\mathbf{3}}$, triiodothyronine; $\mathbf{T}_{\mathbf{4}}$, thyroxine; $\mathbf{T g}$, thyroglobulin; TRAb, TSH receptor autoantibody; TSH, thyroid stimulating hormone; TTR, transthyretin 
ethics review committee of our university hospital, and written parental consent was obtained for all participants. The study abided by the ethical standards of the Declaration of Helsinki (15).

Measurements and blood sampling. Demographic information included maternal age, gestational age, and infants' age, gender, height, weight, BMI, head circumference, chest girth, chronological age (CA), BA, and ratio of BA/CA. Body weight and height were compared with the population curve (data were obtained from the Taiwanese Department of Health) at each visit. Standing height was measured in triplicate using a wall-mounted stadiometer. BMI was calculated by dividing body weight $(\mathrm{kg})$ by the square of height in meters $\left(\mathrm{m}^{2}\right)$. Left hand radiographs were taken, and BA was determined using the method of Greulich and Pyle (16).

In laboratory analysis, thyroid, sex hormone, and growth factor concentrations with respect to PCDD/F exposure level were collected for evaluation. Venous blood samples were obtained from children whose mothers' PCDD/F TEQ levels were available in our database. Blood samples were immediately centrifuged and the serum frozen at $-70^{\circ} \mathrm{C}$ for later analysis of triiodothyronine $\left(\mathrm{T}_{3}\right), \mathrm{T}_{4}, \mathrm{FT}_{4}, \mathrm{TSH}, \mathrm{T}_{3}$ uptake, thyroglobulin $(\mathrm{Tg})$, TSH receptor autoantibody (TRAb) uptake, transthyretin (TTR), IGF-1, IGF binding protein 3 (IGFBP-3), testosterone, and estradiol $\left(\mathrm{E}_{2}\right)$.

$\boldsymbol{T}_{3}, \boldsymbol{T}_{4}, \boldsymbol{F} \boldsymbol{T}_{4}, \boldsymbol{T S H}$, and $\boldsymbol{T}_{3}$ uptake analysis. Serum $\mathrm{T}_{3}, \mathrm{~T}_{4}$, free $\mathrm{T}_{4}$ and $\mathrm{TSH}$ concentrations, and $\mathrm{T}_{3}$ uptake were measured using an automated chemiluminescent immunometric assay (Immulite 2000: Diagnostic Products Corp, Los Angeles, CA). Sensitivities were $19 \mathrm{ng} / \mathrm{dL}$ for $\mathrm{T}_{3}, 0.3 \mu \mathrm{g} / \mathrm{dL}_{\mathrm{L}}$ for $\mathrm{T}_{4}, 0.002$ $\mu \mathrm{IU} / \mathrm{mL}$ for $\mathrm{TSH}$, and $1.6 \mu \mathrm{g} / \mathrm{mL}$ for $\mathrm{T}_{3}$ uptake. Intra- and interassay coefficients of variation (CV) were 4.4 and $5.3 \%$ for $\mathrm{T}_{3} ; 4.6$ and $5.6 \%$ for $\mathrm{T}_{4}$; and 5.1 and $6.4 \%$ for $\mathrm{TSH}$. $\mathrm{FT}_{4} \times \mathrm{TSH}$ values were calculated based on the rationale that, for a normal hypothalamic-pituitary axis, a decrease in $\mathrm{T}_{4}$ feedback inhibition to the hypothalamus resulted leads to TSH secretion by the anterior pituitary.

Tg analysis. Serum Tg levels were measured by immunoradiometric assay (RSR Ltd., Cardiff, UK). Sensitivity was $0.7 \mathrm{ng} / \mathrm{mL}$, and the intra- and interassay CVs were 3.32 and $6.36 \%$.

TRA $\boldsymbol{b}$ analysis. Serum TRAb levels were determined using a commercially available RIA kit (RSR limited, Cardiff, UK). Sensitivity of this assay was 5 units/L. Result was expressed as inhibition of TSH binding index, \% = $100 \times\left(1-\mathrm{B} / \mathrm{B}_{\mathrm{o}}\right)$. Intra- and interassay CVs were 4.15 and $6.02 \%$.

TTR analysis. Serum TTR levels were measured using a kinetic nephelometric method by automated nephelometer (Beckman Coulter, Inc. Array System, Carlsbad, CA). The sensitivity of this assay was $1.17 \mathrm{mg} / \mathrm{dL}$, and the intra- and interassay CV were 2.06 and $1.54 \%$.

IGF-1 and IGFBP-3 analysis. Serum total IGF-I and IGFBP-3 levels were measured after acid extraction using commercial RIA kits (Diagnostic Systems Laboratories, Inc., Webster, TX). The sensitivities were $0.9 \mathrm{mg} / \mathrm{L}$ and $0.01 \mathrm{ng} / \mathrm{mL}$ for IGF-I and IGFBP-3, respectively. The intra- and interassay CV were 7.2 and $9.8 \%$ for IGF-I and 5.8 and $8.2 \%$ for IGFBP-3.

Testosterone analysis. Serum testosterone concentrations were measured by solid-phase, competitive chemiluminescent enzyme immunoassay (Immulite 2000 Advanced Immunoassay System, Siemens Medical Solutions Diagnostics, Deerfield, IL). The sensitivity of this assay was $15 \mathrm{ng} / \mathrm{dL}$. Intra- and interassay CVs were 9.7 and $12.0 \%$.

$\boldsymbol{E}_{2}$ analysis. Serum $\mathrm{E}_{2}$ levels were measured by RIA kit (Diagnostic Systems Laboratories). Sensitivity was $2.2 \mathrm{pg} / \mathrm{mL}$, and intra- and interassay CV were 7.5 and $9.3 \%$.

Statistical analysis. In general analysis, data were expressed as mean \pm SD for continuous variables and frequency with percentage for categorical variables. For comparison of difference in means between groups, a $t$ test was performed for normally distributed data, and the nonparametric Wilcoxon rank sum test was performed for data that were not normally distributed. A multiple regression analysis was performed with a stepwise selection for detecting the factors, which might affect the growth of hormone concentrations by adjusting the maternal age and sex of the infant. All comparisons were considered under a significance level $\alpha=0.05$. All data were analyzed using SAS version 9.0 (SAS Institute Inc., Cary, NC).

\section{RESULTS}

Summary of anthropomorphic measurements by infant gender is presented in Table 1. Significant increases were apparent for each variable between genders at both ages. At year 2, height, weight, BMI, and head circumference were significantly higher in boys than girls. Chest girth was significantly higher in females at this visit. At year 5, there were no between-group differences in height, weight, BMI, head circumference, or chest girth.

Using BA as an indicator of abnormal growth, we compared BA with $\mathrm{CA}$ at both visits. At year 2, children (male and female combined) had a mean BA of $2.14 \pm 0.45$ y $(n=136$; note: BA was not assessed for all subjects at year 2), whereas CA was $2.23 \pm 0.58$ y $(n=194)$. The difference between BA and CA was borderline significant $(p=0.056)$. At year 5, however, mean BA (4.39 $\pm 0.83 \mathrm{y})$ was significantly lower than mean CA $(5.18 \pm 0.19$ y) $(p<0.0001)$ (data not shown). $\mathrm{BA}$ and CA comparisons with respect to gender were conducted as well; no difference was detected at year 2, but males had a significantly lower mean BA than females at year 5 ( $p=$ 0.0058). Significant differences were detected for the mean of ratio of BA and CA with respect to gender at both year 2 and year $5(p=0.0160$ for year $2 ; p<0.0001$ for year 5) (Table 1$)$.

In considering anthropomorphic data and PCDD/Fs exposure levels, high level ( $\geq 15 \mathrm{pg}-\mathrm{TEQ} / \mathrm{g}$ lipid) and low level $(<15 \mathrm{pg}-\mathrm{TEQ} / \mathrm{g}$ lipid $)$ are summarized by age and gender. Overall, height and weight were significantly different between the PCDD/Fs exposure levels at year $2(p=0.0029$ for height and 0.0076 for weight). However, only height remained significant at year 5 . In subgroups (male and female), it was found that height, weight, $\mathrm{CA}$, and $\mathrm{BA}$ were significantly different between the PCDD/Fs exposure levels at year 2 for female $(p=0.0110$ for height, 0.0086 for weight, 0.0218 for CA, and 0.0461 for BA). In contrast, there were no such differences for males. Similarly, there were no significant differences between exposure levels by gender at year 5 (Table 2).

For children who were exposed PCDD/Fs, $\mathrm{T}_{3}$, and IGF-1 concentrations at year 2 were significantly higher in females than males $\left(p=0.0302,0.0434\right.$ for $\mathrm{T}_{3}$ and IGF-1, respec-

Table 1. Summary of anthropomorphic demographics at year 2 and year 5

\begin{tabular}{|c|c|c|c|c|c|c|}
\hline \multirow[b]{2}{*}{ Demographics* } & \multicolumn{3}{|c|}{ Year 2} & \multicolumn{3}{|c|}{ Year 5} \\
\hline & Male $(n=69)$ & Female $(n=67)$ & $p$ & Male $(n=70)$ & Female $(n=79)$ & $p$ \\
\hline Height $(\mathrm{cm})$ & $88.96 \pm 4.33$ & $87.72 \pm 3.56$ & $0.0342 \dagger$ & $111.28 \pm 4.16$ & $110.78 \pm 4.64$ & 0.6000 \\
\hline Weight $(\mathrm{kg})$ & $13.45 \pm 1.91$ & $12.65 \pm 1.66$ & $0.0025 \dagger$ & $20.33 \pm 2.45$ & $20.27 \pm 3.87$ & 0.9393 \\
\hline BMI $\left(\mathrm{kg} / \mathrm{m}^{2}\right)$ & $16.96 \pm 1.64$ & $16.41 \pm 1.57$ & $0.0195 \dagger$ & $18.24 \pm 1.82$ & $17.99 \pm 2.81$ & 0.6281 \\
\hline Head circumference $(\mathrm{cm})$ & $50.85 \pm 2.72$ & $48.18 \pm 1.48$ & $0.0362 \dagger$ & $51.01 \pm 1.50$ & $50.81 \pm 1.60$ & 0.5534 \\
\hline Chest girth $(\mathrm{cm})$ & $48.64 \pm 1.47$ & $49.96 \pm 2.83$ & $0.0293 \dagger$ & $56.95 \pm 2.99$ & $56.53 \pm 4.06$ & 0.5887 \\
\hline $\mathrm{CA}$ & $2.20 \pm 0.28$ & $2.25 \pm 0.27$ & 0.1555 & $5.25 \pm 0.32$ & $5.32 \pm 0.30$ & 0.1815 \\
\hline BA & $2.04 \pm 0.34$ & $2.25 \pm 0.52$ & 0.6419 & $3.99 \pm 0.82$ & $4.84 \pm 0.59$ & 0.0058 \\
\hline $\mathrm{BA} / \mathrm{CA}$ & $0.93 \pm 0.17$ & $1.00 \pm 0.19$ & $0.0160 \dagger$ & $0.76 \pm 0.16$ & $0.91 \pm 0.11$ & $<0.0001 \dagger$ \\
\hline
\end{tabular}

$*$ Demographics are presented as mean $\pm \mathrm{SD}$.

$\dagger p<0.05$ indicates statistically significant difference between genders. 
Table 2. Summary of anthropomorphic variables with respect to PCDD/F exposure levels (low versus high) as determined at year 2 and year 5

\begin{tabular}{|c|c|c|c|c|c|c|c|c|c|}
\hline \multirow[b]{2}{*}{ Demographics* } & \multicolumn{3}{|c|}{ Male } & \multicolumn{3}{|c|}{ Female } & \multicolumn{3}{|c|}{ Overall } \\
\hline & $\begin{array}{c}\text { Low } \dagger \\
(<15.1)\end{array}$ & $\begin{array}{l}\mathrm{High} \dagger \\
(\geq 15.1)\end{array}$ & $p$ & $\begin{array}{c}\text { Low } \dagger \\
(<15.1)\end{array}$ & $\begin{array}{l}\mathrm{High} \dagger \\
(\geq 15.1)\end{array}$ & $p$ & $\begin{array}{c}\text { Low } \dagger \\
(<15.1)\end{array}$ & $\begin{array}{c}\text { High } \dagger \\
(\geq 15.1)\end{array}$ & $p$ \\
\hline \multicolumn{10}{|l|}{ Year 2} \\
\hline$n$ & 14 & 15 & & 21 & 20 & & 35 & 35 & \\
\hline Height $(\mathrm{cm})$ & $89.25 \pm 3.24$ & $91.63 \pm 2.60$ & 0.1531 & $85.77 \pm 2.98$ & $88.71 \pm 2.47$ & $0.0110 \ddagger$ & $86.76 \pm 3.38$ & $89.88 \pm 2.86$ & 0.0029 \\
\hline Weight $(\mathrm{kg})$ & $13.08 \pm 1.53$ & $13.50 \pm 1.20$ & 0.5768 & $12.03 \pm 1.06$ & $13.71 \pm 1.95$ & $0.0086 \ddagger$ & $12.33 \pm 1.27$ & $13.63 \pm 1.65$ & 0.0076 \\
\hline BMI $\left(\mathrm{kg} / \mathrm{m}^{2}\right)$ & $16.36 \pm 0.74$ & $16.09 \pm 1.35$ & 0.6593 & $16.36 \pm 1.18$ & $17.38 \pm 1.96$ & 0.1073 & $16.36 \pm 1.05$ & $16.86 \pm 1.82$ & 0.2931 \\
\hline Head circumference $(\mathrm{cm})$ & $49.17 \pm 1.75$ & $49.75 \pm 1.60$ & 0.5291 & $47.83 \pm 1.23$ & $48.71 \pm 1.47$ & 0.1049 & $48.21 \pm 1.49$ & $49.13 \pm 1.57$ & 0.0641 \\
\hline Chest girth $(\mathrm{cm})$ & $50.50 \pm 1.90$ & $50.75 \pm 1.65$ & 0.7965 & $49.47 \pm 2.90$ & $51.04 \pm 3.75$ & 0.2295 & $49.76 \pm 2.65$ & $50.93 \pm 3.03$ & 0.1975 \\
\hline CA & $2.20 \pm 0.12$ & $2.16 \pm 0.17$ & 0.6382 & $2.09 \pm 0.18$ & $2.24 \pm 0.15$ & $0.0218 \ddagger$ & $2.12 \pm 0.17$ & $2.21 \pm 0.16$ & 0.0804 \\
\hline BA & $2.24 \pm 0.32$ & $2.17 \pm 0.41$ & 0.7472 & $1.99 \pm 0.35$ & $2.40 \pm 0.54$ & $0.0461 \ddagger$ & $2.07 \pm 0.35$ & $2.31 \pm 0.49$ & 0.1143 \\
\hline $\mathrm{BA} / \mathrm{CA}$ & $1.02 \pm 0.14$ & $1.02 \pm 0.23$ & 0.9797 & $0.96 \pm 0.15$ & $1.06 \pm 0.21$ & 0.2021 & $0.98 \pm 0.14$ & $1.04 \pm 0.21$ & 0.3022 \\
\hline \multicolumn{10}{|l|}{ Year 5} \\
\hline$n$ & 6 & 8 & & 15 & 12 & & 21 & 20 & \\
\hline Height $(\mathrm{cm})$ & $110.77 \pm 2.49$ & $113.25 \pm 3.18$ & 0.1409 & $109.45 \pm 4.99$ & $112.75 \pm 5.10$ & 0.1030 & $109.82 \pm 4.40$ & $112.95 \pm 4.34$ & 0.0276 \\
\hline Weight $(\mathrm{kg})$ & $20.58 \pm 3.26$ & $20.54 \pm 1.89$ & 0.9740 & $19.57 \pm 3.24$ & $22.23 \pm 5.19$ & 0.1145 & $19.86 \pm 3.20$ & $21.56 \pm 4.20$ & 0.1520 \\
\hline BMI $\left(\mathrm{kg} / \mathrm{m}^{2}\right)$ & $18.56 \pm 2.70$ & $18.13 \pm 1.56$ & 0.7165 & $17.80 \pm 2.22$ & $19.25 \pm 3.47$ & 0.1973 & $18.01 \pm 2.32$ & $18.81 \pm 2.86$ & 0.3362 \\
\hline Head circumference $(\mathrm{cm})$ & $51.30 \pm 1.76$ & $51.40 \pm 1.68$ & 0.9157 & $50.09 \pm 1.74$ & $51.41 \pm 1.86$ & 0.0685 & $50.43 \pm 1.79$ & $51.41 \pm 1.74$ & 0.0863 \\
\hline Chest girth $(\mathrm{cm})$ & $57.23 \pm 4.08$ & $56.81 \pm 2.93$ & 0.8254 & $55.95 \pm 3.64$ & $58.98 \pm 4.68$ & 0.0695 & $56.32 \pm 3.71$ & $58.12 \pm 4.12$ & 0.1503 \\
\hline $\mathrm{CA}$ & $5.20 \pm 0.12$ & $5.15 \pm 0.17$ & 0.5504 & $5.12 \pm 0.27$ & $5.28 \pm 0.14$ & 0.0626 & $5.15 \pm 0.24$ & $5.23 \pm 0.16$ & 0.1891 \\
\hline BA & $4.17 \pm 0.75$ & $3.81 \pm 0.75$ & 0.4008 & $4.63 \pm 0.69$ & $4.92 \pm 0.56$ & 0.2618 & $4.50 \pm 0.72$ & $4.48 \pm 0.83$ & 0.9188 \\
\hline $\mathrm{BA} / \mathrm{CA}$ & $0.80 \pm 0.15$ & $0.74 \pm 0.16$ & 0.4819 & $0.90 \pm 0.13$ & $0.93 \pm 0.09$ & 0.5828 & $0.88 \pm 0.14$ & $0.85 \pm 0.15$ & 0.6421 \\
\hline
\end{tabular}

$*$ Demographics are presented as mean $\pm \mathrm{SD}$.

$\dagger$ Low and high levels for PCDD/Fs levels were categorized as $<15$, and $\geq 15 \mathrm{pg}-\mathrm{TEQ} / \mathrm{g}$ lipid.

$\ddagger p<0.05$ indicates statistically significant difference between the high- and low-exposure levels for a given gender or overall.

Table 3. Thyroid and sex hormone, and growth factor concentrations with respect to PCDD/F exposure level at year 2

\begin{tabular}{|c|c|c|c|c|c|c|c|c|c|}
\hline \multirow[b]{2}{*}{ Variables* } & \multicolumn{3}{|c|}{ Male } & \multicolumn{3}{|c|}{ Female } & \multicolumn{3}{|c|}{ Overall } \\
\hline & $\begin{array}{c}\text { Low } \dagger \\
(<15.1)\end{array}$ & $\begin{array}{c}\operatorname{High}_{\dagger} \dagger \\
(\geq 15.1)\end{array}$ & $p$ & $\begin{array}{c}\text { Low }{ }^{\dagger} \\
(<15.1)\end{array}$ & $\begin{array}{c}\operatorname{High}_{(} \dagger \\
(\geq 15.1)\end{array}$ & $p$ & $\begin{array}{c}\text { Low } \dagger \\
(<15.1)\end{array}$ & $\begin{array}{c}\mathrm{High}_{\dagger} \dagger \\
(\geq 15.1)\end{array}$ & $p$ \\
\hline$n$ & 14 & 15 & & 21 & 20 & & 35 & 35 & \\
\hline $\mathrm{T}_{3}(\mathrm{ng} / \mathrm{dL})$ & $186.79 \pm 41.05$ & $188.06 \pm 23.89$ & 0.9357 & $154.77 \pm 29.00$ & $176.50 \pm 18.29$ & $0.0236 \ddagger$ & $166.97 \pm 36.72$ & $181.12 \pm 21.04$ & 0.1281 \\
\hline $\mathrm{T}_{4}(\mu \mathrm{g} / \mathrm{dL})$ & $9.83 \pm 2.52$ & $10.33 \pm 2.21$ & 0.6568 & $9.30 \pm 2.79$ & $9.36 \pm 1.71$ & 0.9469 & $9.50 \pm 2.64$ & $9.73 \pm 1.93$ & 0.7310 \\
\hline TSH (mIU/L) & $2.77 \pm 0.91$ & $3.31 \pm 0.86$ & 0.2129 & $2.54 \pm 1.05$ & $3.19 \pm 1.44$ & 0.1857 & $2.63 \pm 0.98$ & $3.24 \pm 1.22$ & 0.0697 \\
\hline $\mathrm{T}_{3}$ uptake $(\%)$ & $26.14 \pm 2.97$ & $26.64 \pm 2.98$ & 0.7816 & $28.37 \pm 4.51$ & $27.97 \pm 1.89$ & 0.7996 & $27.63 \pm 4.10$ & $27.48 \pm 2.36$ & 0.9035 \\
\hline $\mathrm{FT}_{4}(\mathrm{ng} / \mathrm{dL})$ & $1.12 \pm 0.18$ & $1.25 \pm 0.15$ & 0.1291 & $1.13 \pm 0.23$ & $1.20 \pm 0.23$ & 0.4689 & $1.13 \pm 0.21$ & $1.22 \pm 0.20$ & 0.1479 \\
\hline $\mathrm{FT}_{4} \times \mathrm{TSH}$ & $2.78 \pm 0.54$ & $4.11 \pm 1.11$ & $0.0122 \ddagger$ & $2.83 \pm 1.05$ & $3.87 \pm 2.00$ & 0.1155 & $2.81 \pm 0.88$ & $3.97 \pm 1.67$ & $0.0079 \ddagger$ \\
\hline $\mathrm{Tg}(\mathrm{ng} / \mathrm{dL})$ & $42.04 \pm 19.69$ & $35.82 \pm 11.54$ & 0.4409 & $38.05 \pm 18.71$ & $30.13 \pm 11.16$ & 0.2331 & $39.69 \pm 18.61$ & $32.57 \pm 11.41$ & 0.1791 \\
\hline TRAb (\%) & $1.61 \pm 0.59$ & $1.83 \pm 0.81$ & 0.5736 & $1.92 \pm 0.84$ & $1.75 \pm 0.76$ & 0.5928 & $1.81 \pm 0.76$ & $1.78 \pm 0.76$ & 0.8868 \\
\hline TTR (mg/dL) & $12.42 \pm 2.87$ & $12.42 \pm 3.14$ & 0.9978 & $10.81 \pm 2.67$ & $13.87 \pm 3.04$ & $0.0045 \ddagger$ & $11.50 \pm 2.82$ & $13.24 \pm 3.12$ & $0.0298 \ddagger$ \\
\hline $\mathrm{E}_{2}(\mathrm{ng} / \mathrm{dL})$ & $7.64 \pm 1.60$ & $8.44 \pm 1.75$ & 0.3539 & $8.48 \pm 1.10$ & $9.09 \pm 2.31$ & 0.3684 & $8.19 \pm 1.32$ & $8.83 \pm 2.09$ & 0.2132 \\
\hline Testosterone (ng/dL) & $3.81 \pm 1.52$ & $3.42 \pm 1.74$ & 0.6224 & $3.62 \pm 1.61$ & $3.31 \pm 1.24$ & 0.5618 & $3.70 \pm 1.54$ & $3.35 \pm 1.43$ & 0.4371 \\
\hline IGF-1 (ng/mL) & $94.73 \pm 34.70$ & $131.73 \pm 39.55$ & 0.0596 & $133.69 \pm 80.20$ & $168.89 \pm 69.39$ & 0.2515 & $118.11 \pm 67.46$ & $153.69 \pm 60.80$ & 0.0797 \\
\hline IGFBP-3 (ng/mL) & $2.80 \pm 0.54$ & $3.18 \pm 0.64$ & 0.1921 & $3.79 \pm 2.49$ & $3.40 \pm 0.68$ & 0.5842 & $3.41 \pm 2.02$ & $3.31 \pm 0.66$ & 0.8230 \\
\hline
\end{tabular}

* Variables including thyroid and sex hormone, and growth factor concentrations were presented as the mean \pm SD.

$\dagger$ Low and high levels for PCDD/Fs levels were categorized as $<15$, and $\geq 15 \mathrm{pg}-\mathrm{TEQ} / \mathrm{g}$ lipid.

$\$ p<0.05$ indicates statistically significant difference between the high and low exposure levels for a given gender or overall.

tively). At year 5, $\mathrm{FT}_{4}$ was significantly higher in males ( $p=$ $0.0105)$, whereas IGF-1 levels were significantly higher in females $(p=0.0433$; data not shown).

The thyroid hormone, sex hormone, and growth factor concentrations with respect to $\mathrm{PCDD} / \mathrm{Fs}$ exposure levels, high level ( $\geq 15 \mathrm{pg}-\mathrm{TEQ} / \mathrm{g}$ lipid) and low level $(<15 \mathrm{pg}-\mathrm{TEQ} / \mathrm{g}$ lipid) were summarized by gender for year 2 and year 5 , respectively (Table 3 and Table 4). Overall, $\mathrm{FT}_{4} \times \mathrm{TSH}$, TTR levels at year 2 and $\mathrm{T}_{3}$, IGF-1 at year 5 were significantly higher in the group with high dioxin/PCB exposure level than the group with low exposure level $(p=0.0079,0.0298$ for $\mathrm{FT}_{4} \times \mathrm{TSH}$ and TTR at year 2, respectively; $p=0.0379$,
0.0243 for $\mathrm{T}_{3}$ and IGF-1 at year 5 , respectively). In females, only $\mathrm{T}_{3}$ and TTR concentrations at year 2 were significantly higher in the high PCDD/Fs exposure level than the low level ( $p=0.0236,0.0045$ for $\mathrm{T}_{3}$ and TTR, respectively), whereas there was no significant difference detected in concentrations at year 5. In males, $\mathrm{FT}_{4} \times \mathrm{TSH}$ at year 2 and IGF-1 at year 5 were significantly higher in the high PCDD/Fs exposure level than the low level $\left(p=0.0122\right.$ for $\mathrm{FT}_{4} \times \mathrm{TSH}$ at year 2 , and $p=0.0076$ for IGF-1 at year 5).

Results of multiple linear regression analysis adjusting for maternal age indicated that, overall, only $\mathrm{FT}_{4} \times \mathrm{TSH}$ at year 2 and IGF-1 at year 5 were significantly higher in the group 
Table 4. Thyroid hormone, sex hormone, and growth factor concentrations with respect to PCDD/F exposure level at year 5

\begin{tabular}{|c|c|c|c|c|c|c|c|c|c|}
\hline \multirow[b]{2}{*}{ Variables* } & \multicolumn{3}{|c|}{ Male } & \multicolumn{3}{|c|}{ Female } & \multicolumn{3}{|c|}{ Overall } \\
\hline & $\begin{array}{c}\text { Low } \dagger \\
(<15.1)\end{array}$ & $\begin{array}{c}\mathrm{High}_{\dagger} \dagger \\
(\geq 15.1)\end{array}$ & $p$ & $\begin{array}{c}\text { Low } \dagger \\
(<15.1)\end{array}$ & $\begin{array}{c}\mathrm{High}_{\dagger} \dagger \\
(\geq 15.1)\end{array}$ & $p$ & $\begin{array}{c}\text { Low } \dagger \\
(<15.1)\end{array}$ & $\begin{array}{c}\mathrm{High}_{\dagger} \dagger \\
(\geq 15.1)\end{array}$ & $p$ \\
\hline$n$ & 6 & 8 & & 15 & 12 & & 21 & 20 & \\
\hline $\mathrm{T}_{3}(\mathrm{ng} / \mathrm{dL})$ & $144.30 \pm 11.74$ & $151.88 \pm 18.03$ & 0.3897 & $135.50 \pm 15.67$ & $146.36 \pm 15.80$ & 0.0868 & $138.01 \pm 14.93$ & $148.57 \pm 16.49$ & $0.0379 \ddagger$ \\
\hline $\mathrm{T}_{4}(\mu \mathrm{g} / \mathrm{dL})$ & $7.98 \pm 2.21$ & $8.78 \pm 2.01$ & 0.4975 & $8.29 \pm 2.02$ & $8.33 \pm 1.95$ & 0.9641 & $8.20 \pm 2.02$ & $8.51 \pm 1.94$ & 0.6275 \\
\hline TSH (mIU/L) & $2.60 \pm 1.34$ & $2.99 \pm 0.95$ & 0.5327 & $2.73 \pm 1.72$ & $2.86 \pm 2.22$ & 0.8700 & $2.69 \pm 1.59$ & $2.91 \pm 1.78$ & 0.6843 \\
\hline $\mathrm{T}_{3}$ uptake $(\%)$ & $26.72 \pm 2.21$ & $26.84 \pm 2.34$ & 0.9237 & $27.13 \pm 3.37$ & $26.09 \pm 2.50$ & 0.3824 & $27.01 \pm 3.04$ & $26.39 \pm 2.40$ & 0.4722 \\
\hline $\mathrm{FT}_{4}(\mathrm{ng} / \mathrm{dL})$ & $1.29 \pm 0.25$ & $1.33 \pm 0.22$ & 0.7665 & $1.24 \pm 0.28$ & $1.11 \pm 0.14$ & 0.1166 & $1.26 \pm 0.27$ & $1.20 \pm 0.21$ & 0.4172 \\
\hline $\mathrm{FT}_{4} \times \mathrm{TSH}$ & $3.47 \pm 2.32$ & $4.12 \pm 1.76$ & 0.5643 & $3.38 \pm 2.58$ & $3.13 \pm 2.24$ & 0.7875 & $3.41 \pm 2.45$ & $3.52 \pm 2.07$ & 0.8735 \\
\hline $\operatorname{Tg}(\mathrm{ng} / \mathrm{dL})$ & $20.88 \pm 7.62$ & $24.41 \pm 3.58$ & 0.3129 & $24.32 \pm 5.37$ & $25.66 \pm 4.19$ & 0.5536 & $23.51 \pm 5.90$ & $25.08 \pm 3.83$ & 0.3852 \\
\hline $\mathrm{E}_{2}(\mathrm{ng} / \mathrm{dL})$ & $6.74 \pm 2.32$ & $6.47 \pm 1.72$ & 0.8085 & $7.51 \pm 2.30$ & $5.93 \pm 1.62$ & 0.0563 & $7.29 \pm 2.28$ & $6.15 \pm 1.64$ & 0.0748 \\
\hline Testosterone (ng/dL) & $6.60 \pm 1.81$ & $6.27 \pm 1.53$ & 0.7158 & $6.58 \pm 1.59$ & $6.72 \pm 1.81$ & 0.8319 & $6.59 \pm 1.61$ & $6.54 \pm 1.68$ & 0.9281 \\
\hline IGF-1 (ng/mL) & $161.22 \pm 57.79$ & $263.85 \pm 60.43$ & $0.0076 \ddagger$ & $252.96 \pm 122.61$ & $334.28 \pm 114.97$ & 0.0907 & $226.75 \pm 114.73$ & $306.11 \pm 101.25$ & $0.0243 \ddagger$ \\
\hline IGFBP-3 (ng/mL) & $4.79 \pm 1.38$ & $5.38 \pm 0.98$ & 0.3678 & $5.06 \pm 0.98$ & $5.46 \pm 1.17$ & 0.3412 & $4.98 \pm 1.08$ & $5.43 \pm 1.07$ & 0.1928 \\
\hline
\end{tabular}

* Variables including thyroid and sex hormone, and growth factor concentrations were presented as the mean $\pm \mathrm{SD}$.

$\dagger$ Low and high levels for PCDD/Fs levels were categorized as $<15$ and $\geq 15$ pg-TEQ/g lipid.

$\ddagger p<0.05$ indicated a statistically significant difference between the high and low exposure levels for a given gender or overall.

Table 5. Stepwise multiple linear regression models for selection in thyroid hormone concentrations with respect to $P C D D / F$ exposure level at year 2

\begin{tabular}{crc}
\hline \multicolumn{1}{c}{ Model $^{*}$} & Estimate $\pm \mathrm{SE}$ & $p$-Value \\
\hline $\mathrm{T}_{3}, R^{2}=0.3698$ & & \\
$\mathrm{PCDD} / \mathrm{F}$ & $12.59 \pm 4.75$ & $0.0133 \ddagger$ \\
$(\mathrm{PCDD} / \mathrm{F})^{2}$ & $-0.32 \pm 0.13$ & $0.0201 \ddagger$ \\
$\mathrm{TSH} \dagger, R^{2}=0.2221$ & & \\
$\quad$ Maternal age & $-0.05 \pm 0.07$ & $0.0238 \ddagger$ \\
PCDD/F & $0.05 \pm 0.02$ & $0.0188 \ddagger$ \\
$\mathrm{FT}_{4} \times \mathrm{TSH} \dagger, R^{2}=0.2880$ & & \\
$\quad$ Maternal age & $-0.07 \pm 0.03$ & $0.0195 \ddagger$ \\
PCDD/F & $0.06 \pm 0.02$ & $0.0089 \ddagger$ \\
$\mathrm{TTR}, R^{2}=0.0619$ & & \\
PCDD/F & $0.12 \pm 0.08$ & 0.1720 \\
\hline
\end{tabular}

* Model as performed adjusting infants' gender.

$\dagger \mathrm{TSH}$ and $\mathrm{FT}_{4} \times \mathrm{TSH}$ were $\log$ transformed to meet regression assumptions. $\ddagger p<0.05$ indicates a statistically significant relationship.

with high $\mathrm{PCDD} / \mathrm{Fs}$ exposure level than the group with low level exposure $\left(p=0.0002\right.$ for $\mathrm{FT}_{4} \times \mathrm{TSH}$ at year $2 ; p=$ 0.0243 for IGF-1 at year 5). In males, $\mathrm{FT}_{4} \times \mathrm{TSH}$ at year 2 and IGF-1 at year 5 were still significantly higher in the group with high PCDD/Fs exposure level than the group with low exposure level $\left(p=0.0072\right.$ for $\mathrm{FT}_{4} \times \mathrm{TSH}$ at year 2 , and $p=$ 0.0228 for IGF-1 at year 5). In females, TTR was still significantly higher in the high PCDD/Fs exposure level than the low level at year $2 ; \mathrm{FT}_{4} \times \mathrm{TSH}\left(\right.$ instead of $\mathrm{T}_{3}$ ) was significantly higher in the high PCDD/Fs exposure level. There was no significant difference in hormone levels at year 5 (data not shown).

Table 5 shows the results of the stepwise multiple linear regression analysis of selection of thyroid hormone concentrations at first follow-up (year 2) with respect to $\mathrm{PCDD} / \mathrm{Fs}$ exposure level, considering mother's age, parity at first follow-up, and the quadratic form of PCDD/Fs. There were significantly positive correlations among $\mathrm{T}_{3}, \mathrm{TSH}, \mathrm{FT}_{4} \times$ TSH, and TTR concentrations and the level of PCDD/Fs exposure. Both TSH and $\mathrm{FT}_{4} \times \mathrm{TSH}$ concentrations were significantly negatively correlated with maternal age. There was also a negative correlation between $T_{3}$ levels and the

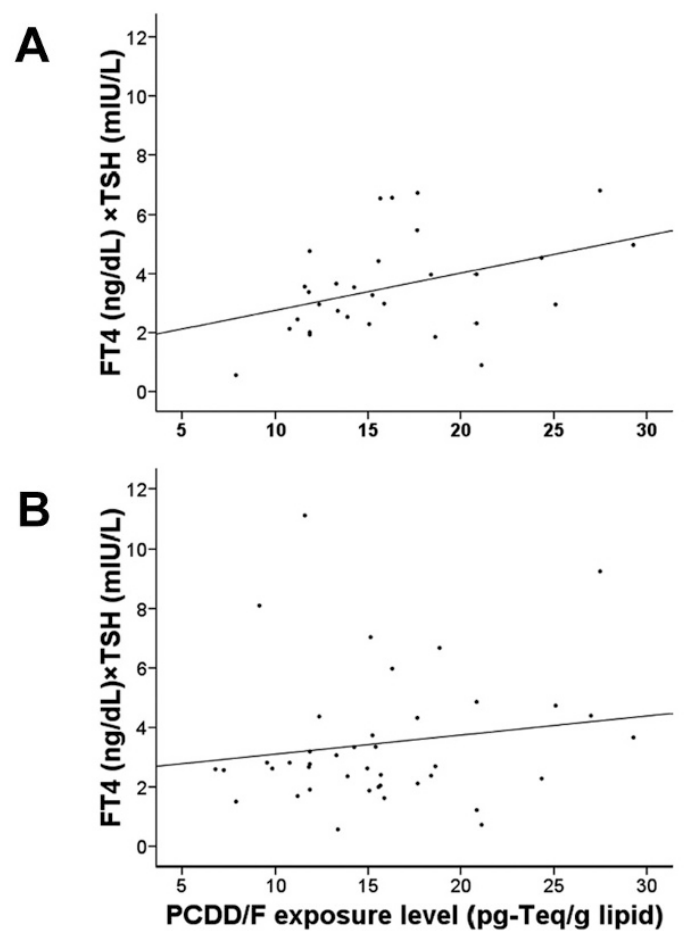

Figure 1. PCDD/F exposure level in relation to $\mathrm{FT}_{4} \times \mathrm{TSH}$ level. By simple linear model: $(A)$ for $2 \mathrm{y}$ preschool data, $\mathrm{FT}_{4} \times \mathrm{TSH}=1.49+0.12 \times$ $(\mathrm{PCDD} / \mathrm{F}), R^{2}=0.1551, p=0.0313 ;(B)$ for 5 -y follow-up, $\mathrm{FT}_{4} \times \mathrm{TSH}=$ $2.46+0.06 \times(\mathrm{PCDD} / \mathrm{F}), R^{2}=0.0247, p=0.3266$.

quadratic form of PCDD/Fs. The significant relation between $\mathrm{FT}_{4} \times$ TSHlevels and PCDD/Fs exposure level at year 2 was further demonstrated by multiple linear model (Fig. 1).

\section{DISCUSSION}

PCDD/Fs are transferred from mother to the fetus through the placenta and to infants via breast milk (10-12). Therefore, maternal exposure to these endocrine disruptors may adversely impact fetal/infant development and functioning. In this study, we performed follow-up examinations of infants/ children previously demonstrated to be exposed in utero to 
varying levels of PCDD/F (13). Our findings indicate that children with higher levels of exposure to these environmental contaminants in utero were significantly taller and heavier and had higher serum level of several thyroid hormones $\left(\mathrm{T}_{3}, \mathrm{FT}_{4}\right.$ $\times$ TSH, and TTR) and growth hormone (IGF-1) at the preschool ages. Whether there is a cause-effect relationship between growth and hormone remains to be studied.

Females seem to be more sensitive than males to PCDD/Fs exposure. Females exposed to PCDD/Fs in utero were significantly taller and heavier and had higher BA/CA ratio than male at both follow-up visits. We also noted the intriguing finding of significantly higher T3 and IGF-1 levels in females than males at year 2. IGF-1 remained higher at year 5 visit. Females exposed to high levels of PCDD/Fs were significantly taller and heavier and had higher BA and CA those with low exposure at year 2 follow-up visits. Similarly, we observed a significant correlation between exposure level of PCDD/Fs and thyroid hormones $\left(\mathrm{T}_{3}, \mathrm{TTR}\right)$ in females at the year 2 follow-up visit. There was no significant correlation between PCDD/Fs exposure level and growth or hormone level in girls at the year 5 follow-up, suggesting that fetal exposure to $\mathrm{PCDD} / \mathrm{Fs}$ can stimulate growth at early ages but it may become less apparent with increasing age. For males, exposure level did not significantly affect growth, but level of PCDD/Fs exposure in utero had a significant correlation with FT4 $\times$ TSH at both year 2 and year 5 .

Correlation between PCDD/Fs exposure level in utero and hormone level during preschool ages was further confirmed using a stepwise multiple linear regression analysis. The results suggested that there were significantly positive correlations between $\mathrm{T}_{3}$, TSH, $\mathrm{FT}_{4} \times \mathrm{TSH}$, and TTR concentrations and the level of PCDD/Fs exposure at year 2 follow-up. The finding of higher $\mathrm{FT}_{4} \times \mathrm{TSH}$ values in the high exposure group suggested that $\mathrm{T}_{4}$ feedback inhibition of TSH secretion was impaired in both males and females exposed to high levels of PCDD/Fs.

A number of previous studies have noted altered thyroid functioning in dioxin/PCB exposed infants. The major finding of this study was the demonstration of significant positive correlations between $\mathrm{T}_{3}, \mathrm{TSH}, \mathrm{FT}_{4} \times \mathrm{TSH}$, and TTR concentrations and the level of PCDD/Fs exposure at year 2 followup. Pluim et al. $(9,17)$ also reported that term infants exposed to upper-median dioxin levels (as measured in breast milk) had increased plasma $\mathrm{T}_{4}$ and $\mathrm{TBG}$ concentrations. Sauer et al. (18) investigated the growth and development of infants in highly industrialized western countries; they found a higher TSH at higher levels of dioxin exposure.

We have noted in a previous publication that $\mathrm{FT}_{4} \times \mathrm{TSH}$ decreased with increasing concentrations of non-ortho PCB exposure levels in infants, indicating a lack of effective $\mathrm{FT}_{4}$ feedback to stimulate the hypothalamus (13). It suggested that dioxin/PCB levels in utero may have differential effect in neonates and children 2-3 y of age. The reason for the difference between two developmental stages is unclear and warrants further investigation. Interestingly, there was no significant correlation between PCDD/Fs exposure level and hormone level at year 5 visit after stepwise multiple linear regression analysis. Mazhitova et al. (14) found that none of the schoolchildren (aged 7.5-15 y), whose serum level of PCBs was two to four times higher than healthy Stockholm children, had any impairment of thyroid function, as revealed by the plasma levels of TSH and thyroxine; there was no relation between thyroid hormone status and the blood lipid levels of PCBs, hexachlorocyclohexane, and DDT. Findings from our previous study suggest that there is a negative correlation between thyroid hormone levels and maternal age (13). In accordance with that study, we also observed a significant negative correlation between $\mathrm{TSH}$ and $\mathrm{FT}_{4} \times \mathrm{TSH}$ concentrations and maternal age, suggesting that children born to older mothers tend to have lower levels of thyroid hormone. It is apparent that further studies are needed to explore in more detail how changes in IGF-1, $\mathrm{T}_{3}, \mathrm{TTR}$, and $\mathrm{FT}_{4} \times \mathrm{TSH}$ correlate with growth and development in infants/children following in utero exposure to PCDD/Fs. Exposure level did not affect $E_{2}$ and testosterone at the early stage of life. In the future, we plan to closely follow this cohort of children and investigate possible differences in the levels of hormones involved in regulating sexual development (i.e. $\mathrm{E}_{2}$ and testosterone) with respect to exposure level.

The same cohort of children will be continuously followed up though school age and puberty. It will be particularly interesting to know if material exposure level and blood levels of PCDD/Fs have any effect on hormone status and development during puberty.

In conclusion, the results of this first complete follow-up study of preschool children exposed to PCDD/Fs in utero indicate that such exposure can significantly affect growth and development. Serum levels of several thyroid hormones showed positive correlation with the level of PCDD/Fs exposure in utero. Age-related changes were also observed. A number of gender-specific differences were detected with regard to growth, basal hormone concentrations, and PCDD/Fs exposure level. These findings emphasize that in utero exposure to PCDD/Fs can affect the growth and development of preschool age children.

Acknowledgments. We thank Ms Hsiao-Yen Chen for specimen collections.

\section{REFERENCES}

1. Liem AK, Furst P, Rappe C 2000 Exposure of populations to dioxins and related compounds. Food Addit Contam 17:241-259

2. Yamashita F, Hayashi M 1985 Fetal PCB syndrome: clinical features, intrauterine growth retardation and possible alteration in calcium metabolism. Environ Health Perspect 59:41-45

3. Rylander L, Stromberg U, Dyremark E, Ostman C, Nilsson-Ehle P, Hagmar L 1998 Polychlorinated biphenyls in blood plasma among Swedish female fish consumers in relation to low birth weight. Am J Epidemiol 147:493-502

4. Buck GM, Sever LE, Mendola P, Zielezny M, Vena JE 1997 Consumption of contaminated sport fish from Lake Ontario and time-to-pregnancy. New York State Angler Cohort. Am J Epidemiol 146:949-954

5. Guo YL, Hsu PC, Hsu CC, Lambert GH 2000 Semen quality after prenatal exposure to polychlorinated biphenyls and dibenzofurans. Lancet 356:1240-1241

6. Schecter A, Olson JR 1997 Cancer risk assessment using blood dioxin levels and daily dietary TEQ intake in general populations of industrial and non-industrial countries. Chemosphere 34:1569-1577

7. Demers A, Ayotte P, Brisson J, Dodin S, Robert J, Dewailly E 2002 Plasma concentrations of polychlorinated biphenyls and the risk of breast cancer: a congener-specific analysis. Am J Epidemiol 155:629-635

8. Jacobson JL, Jacobson SW 2001 Developmental effects of PCBs in the fish eater cohort studies. In: Robertson LW, Hansen LG (eds) PCBs: Recent Advances in Environmental Toxicology and Health Effects. The University Press of Kentucky, Lexington, pp 127-128 
9. Pluim HJ, De Vijlder JJ, Olie K, Kok JH, Vulsma T, van Tijn DA, van der Slikke JW, Koppe JG 1993 Effects of pre- and postnatal exposure to chlorinated dioxins and furans on human neonatal thyroid hormone concentrations. Environ Health Perspect 101:504-508

10. Wang SL, Lin CY, Guo YL, Lin LY, Chou WL, Chang LW 2004 Infant exposure to polychlorinated dibenzo-p-dioxins, dibenzofurans and biphenyls (PCDD/Fs, $\mathrm{PCBs}$ )-correlation between prenatal and postnatal exposure. Chemosphere $54: 1459-1473$

11. Chao HR, Wang SL, Lee CC, Yu HY, Lu YK, Papke O 2004 Level of polychlorinated dibenzo-p-dioxins, dibenzofurans and biphenyls (PCDD/Fs, PCBs) in human milk and the input to infant body burden. Food Chem Toxicol 42:1299-1308

12. Ma HW, Lai YL, Chan CC 2002 Transfer of dioxin risk between nine major municipal waste incinerators in Taiwan. Environ Int 28:103-110

13. Wang SL, Su PH, Jong SB, Guo YL, Chou WL, Päpke O 2005 In utero exposure to dioxins and polychlorinated biphenyls and its relations to thyroid function and growth hormone in newborns. Environ Health Perspect 113:1645-1650
14. Mazhitova Z, Jensen S, Ritzén M, Zetterström R 1998 Chlorinated contaminants, growth and thyroid function in schoolchildren from the Aral Sea region in Kazakhstan. Acta Paediatr 87:991-995

15. 2000 World Medical Association Declaration of Helsinki: ethical principles for medical research involving human subjects. JAMA 284:3043-3045

16. Greulich WW, Pyle SI, Waterhouse AM 1971 A radiographic standard of reference for the growing hand and wrist. Chicago: Case Western Reserve University

17. Pluim HJ, Koppe JG, Olie K, Vd Slikke JW, Kok JH, Vulsma T, Van Tijn D, De Vijlder JJ 1992 Effects of dioxins on thyroid function in newborn babies. Lancet 339:1303

18. Sauer PJ, Huisman M, Koopman-Esseboom C, Morse DC, Smits-van Prooije AE, van de Berg KJ, Tuinstra LG, van der Paauw CG, Boersma ER, Weisglas-Kuperus N, Lammers JH, Kulig BM, Brouwer A 1994 Effects of polychlorinated biphenyls (PCBs) and dioxins on growth and development. Hum Exp Toxicol 13:900906 